Maquilón Sánchez, J.J., Mirete Ruiz, A.B. \& Avilés Olmos, M. (2017). La Realidad Aumentada (RA). Recursos y propuestas para la innovación educativa. Revista Electrónica Interuniversitaria de Formación del Profesorado, 20(2), 183-203.

\title{
La Realidad Aumentada (RA). Recursos y propuestas para la innovación educativa
}

Javier J. Maquilón Sánchez, Ana Belén Mirete Ruiz, Marina Avilés Olmos

Universidad de Murcia

\section{Resumen}

Este artículo contiene una propuesta de experiencia práctica de enseñanza-aprendizaje, basada en la realidad aumentada (RA) como recurso didáctico innovador. RA es tanto un concepto como una tecnología 3D interactiva actualmente en desarrollo. Por este motivo, en primer lugar se ha expuesto, por un lado, qué es la RA y, por otro lado, cuáles son las variantes tecnológicas actuales principales. Seguidamente, se ha hecho un análisis reflexivo de varios aspectos, que el docente ha de considerar respecto a la RA como tecnología aplicada a la educación, destacando la aportación innovadora de la RA como recurso didáctico. Finalmente se presenta el diseño y la elaboración de la actividad, tipo webquest que incluye un blog de aula.

\section{Palabras clave}

Realidad aumentada (RA); recurso didáctico innovador; webquest; blog de aula.

\section{Augmented Reality (AR). Resources and proposals for teaching innovation}

\section{Abstract}

This article contains a proposal of innovative learning-teaching practical experience, based on augmented reality (AR) as didactic resource. AR is as well a concept, as a 3D interactive technology, which is currently enroot. For this reason, first of all we expose a definition of

\section{Contacto:}

Javier J. Maquilón Sánchez. jjmaqui@um.es. Departamento de Métodos de Investigación y Diagnóstico en Educación. Universidad de Murcia (España). 
what does the concept $A R$ mean and which are the main current varieties of the AR technology. Next, we present a reflective analysis of several aspects, which must be considered by the teacher, regarding the AR as applied technology in education and we highlight the AR's innovation key point in learning contexts, due to the real time interaction, that the pupil can establish with the learning content, this one modeled as $3 \mathrm{D}$ virtual objects. Finally, we show the design of the AR based didactic activity, that follows a webquest model and includes a teaching blog.

\section{Key words}

Augmented reality (AR); innovative teaching resource; webquest; teaching blog.

\section{Introducción}

Las tendencias actuales en educación se aúnan al reconocer la importancia de la integración de enseñanzas-aprendizajes significativos, aprendizaje integral para la vida, metodologías activas, hacer y comprender, uso de las Tecnologías de la Información y la Comunicación (TIC), Tecnologías para el Aprendizaje y el Conocimiento (TAC).

Al mismo tiempo se ha desarrollado un conglomerado extenso de tecnologías específicas en diferentes áreas cuyo currículo digital invita a una integración de innovaciones tecnológicas en las "clásicas" asignaturas de enseñanza. La aceptación popular masiva de la importancia de la imagen y la comunicación audiovisual, común ya en el día a día, se ha desarrollado muy rápidamente gracias a las TIC y a los avances en informática. Hacer y ver a diario fotografías y videos digitales está al alcance de todos. Esta producción masiva de material de comunicación y de expresión visual tiene un gran potencial didáctico y de “comunicación conscientemente diseñada". Entre las tecnologías actuales en desarrollo se encuentra la Realidad Aumentada (RA), la cual está abriendo un ámbito de aplicación innovador en el campo de la educación (Johnson \& otros, 2016).

La investigación en el ámbito de la RA aplicada al proceso de enseñanza-aprendizaje como metodología activa, encuentra su interés en sus carácterísticas innatas, ya que se basa en (figura 1)(Agudelo Toro, 2005):

- $\quad$ Representaciones de objetos 3D

- Objetos virtuales añadidos artificialmente por medios tecnológicos a la realidad del entorno material, por medio de un dispositivo display.

- $\quad$ La interacción con el observador

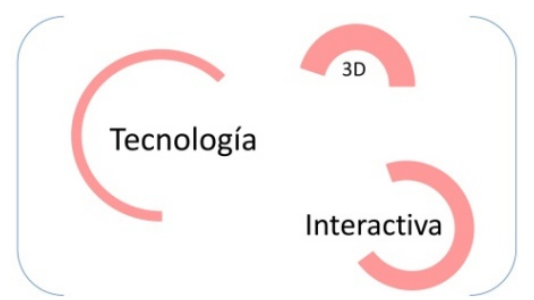

Figura 1. Características específicas de la RA. (Agudelo Toro, 2005) 
Además del carácter innovador singular de esta tecnología por los motivos previamente descritos, la RA se propone no de modo aislado sino dentro de un conjunto de recursos basados en las TIC.

El docente actual debe guiar en el desarrollo de un proceso de aprendizaje que, junto a la web 3.0, ha de facilitar que el alumno se forme en el mundo de la identidad digital, los perfiles personales online, los social media,... (Díaz Barriga, 2013) (Cubillo Arribas, 2014). La realidad aumentada es un recurso didáctico que se puede integrar con este fin en educación. Por otro lado la realidad aumentada ha demostrado tener la capacidad de proporcionar al alumnado una mejor comprensión de conceptos como el espacio tridimensional, formas geométricas, determinadas áreas matemático-geométricas (Cubillo Arribas, 2014) (Cubillo, Martín, Castro \& Colmenar, 2014) (De la Torre, Martin-Dorta, Saorín, Carbonell \& Contero, 2013) (Flores, Domínguez \& Rodríguez, 2010).

En este trabajo presentamos la RA como recurso didáctico innovador en el proceso de enseñanza aprendizaje, proponiendo un ejemplo de aplicación práctica en el aula.

La experiencia nos ha llevado a la elaboración de recursos específicos de RA para el aprendizaje de la realidad aumentada como recurso didáctico para la innovación educativa. Ahora bien, conscientes de las dificultades que encuentran los docentes en la implementación de recursos innovadores de corte tecnológico, se ha elaborado una página web desde la cual facilitar el desarrollo en el aula de este tipo de materiales.

Por este motivo, este trabajo dedica una parte de su desarrollo a la presentación de los materiales didácticos creados para realizar una experiencia didáctica basada en la aplicación de RA, los cuales podrán servir de guía y orientación para aquellos profesores interesados en la implementación de la RA en el aula, independientemente del área en la que desarrollen su labor docente.

\section{Qué es la realidad aumentada}

Para poder hacer una propuesta innovadora con RA el docente debe tener unas nociones básicas de esta tecnología: ¿Qué es la RA? ¿Cuáles son sus características propias? ¿Qué variantes principales hay de la tecnología RA? ¿Cuáles son los entornos más habituales actualmente en la experiencia RA?

La RA es una de las diez tecnologías en desarrollo con mayor potencial pedagógico desde 2008 (Gardner Research, 2008). El término realidad aumentada, abreviado RA, comprende la ampliación artificial de la percepción de la realidad, por medio de información virtual (figura 3). Dicha información virtual es generada con técnicas asistidas por ordenador y representada mediante los componentes tecnológicos específicos. La RA puede abordar todos los sentidos humanos de la percepción, sin embargo la variación de RA más extendida comúnmente es la representación de información virtual visual añadida al entorno real (figuras 2 y 4 ). 


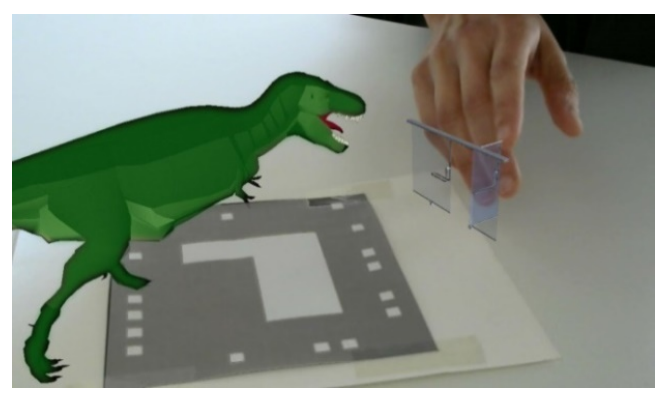

Figura 2. Definición de RA.


Figura 3. Ejemplo de RA.
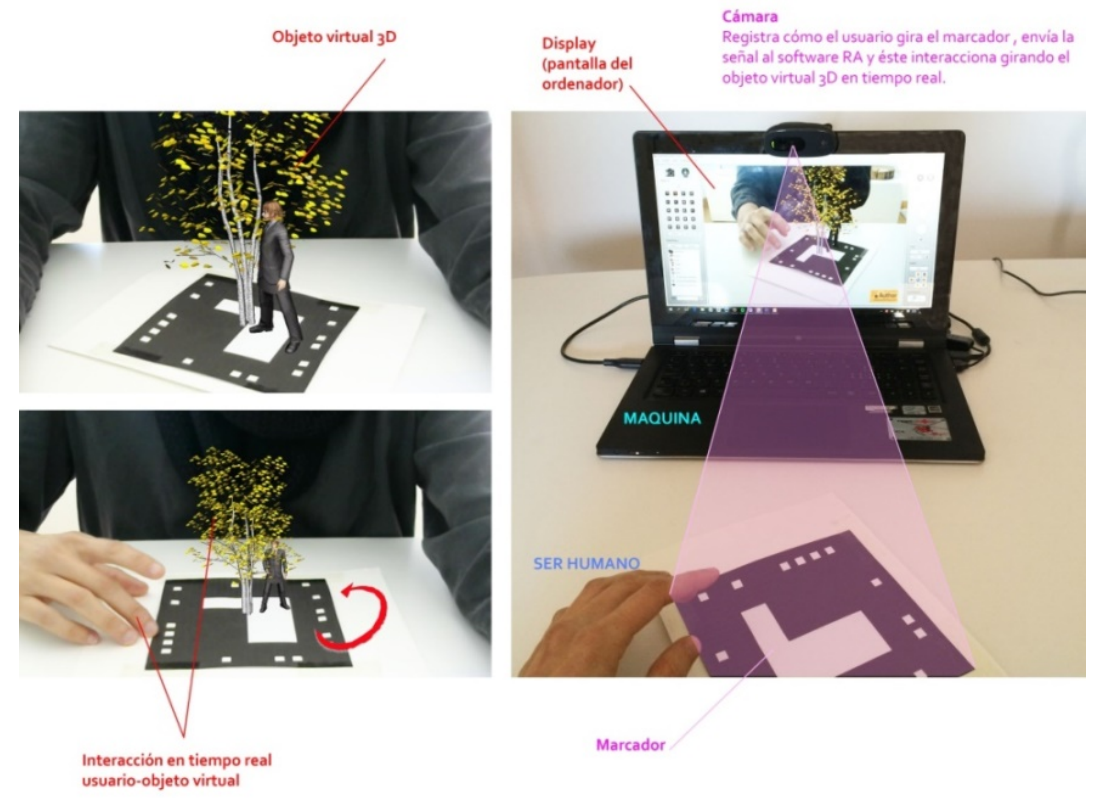

Figura 4. El "making off" de una modalidad de RA. 


\section{La tecnología RA y sus variantes principales}

A partir de este concepto fundamental de RA los avances tecnológicos han generado un desarrollo de variantes muy extenso de esta tecnología. Enumerar, describir o explicar la casuística de todas las variantes de este sistema, queda fuera de los objetivos de esta propuesta. Además, en la literatura científica, cada autor, según el aspecto principal de su investigación, realiza clasificaciones especificas distintas.

El funcionamiento básico de esta tecnología se corresponde de modo global con el siguiente esquema (figura 5):

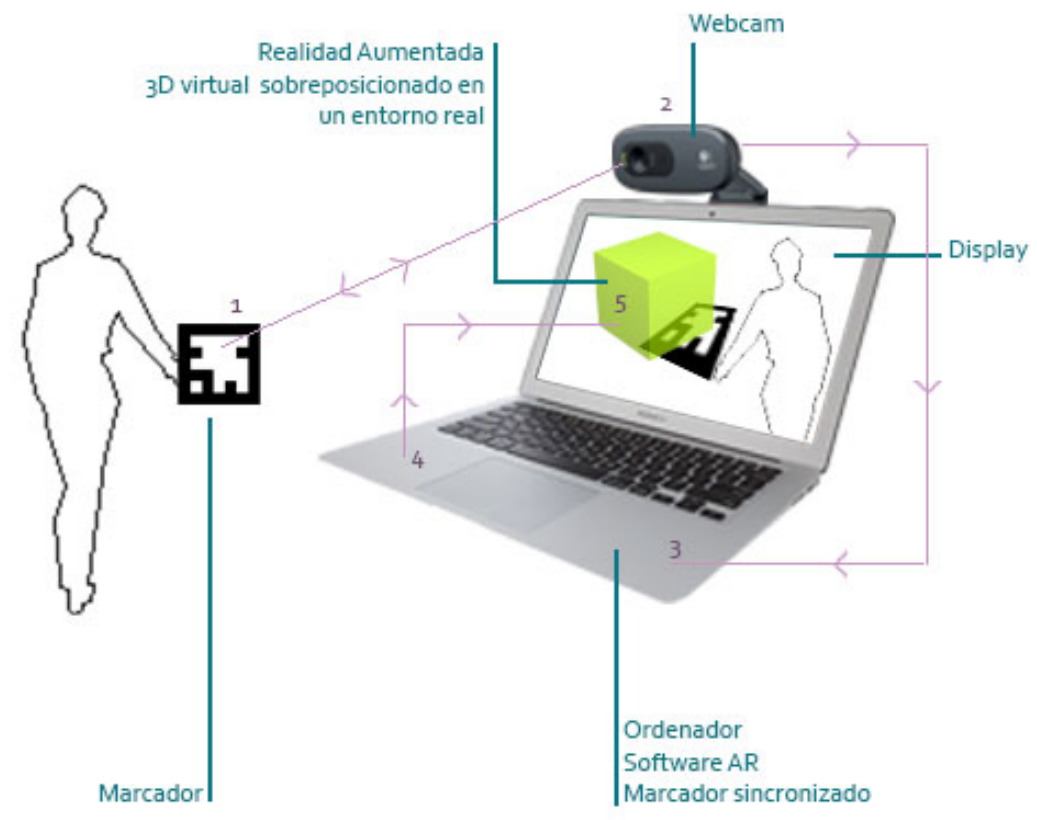

Figura 5. Esquema de funcionamiento básico de la RA.

Para orientar al docente interesado en aplicar la tecnología RA en su docencia y para abrir un horizonte amplio de posibles aplicaciones, a continuación se presenta una explicación esquemática de las variantes principales, según se considere:

- $\quad$ El contexto realidad-virtualidad (figura 6) (Agudelo Toro, 2005) (Billinghurst \& Kato, Collaborative Augmented Reality, 2016)

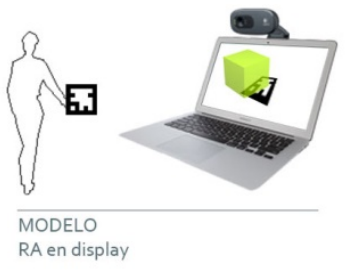

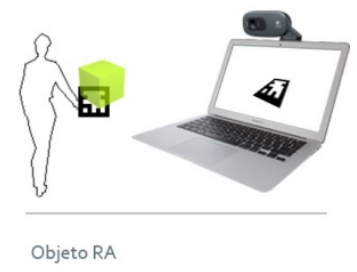

Figura 6. Modelos de contexto RA.




- $\quad$ El componente display (figura 7) (Billinghurst, Mobile AR Lecture 2 - Technology, 2016)

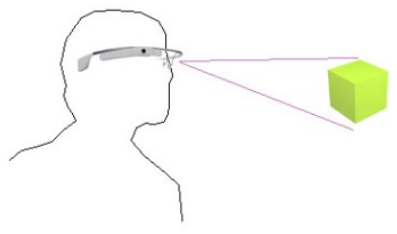

DISPLAY

Acoplado en la cabeza



Acoplado en el cuerpo (la mano)



Espacial

Figura 7. Variantes del componente display en RA.

- La tipología de las interacciones (Billinghurst, Mobile AR Lecture 2 - Technology, 2016)

- Con Navegador (figura 8), respondiendo la interacción a modelos de georeferenciación y creando respuestas fijas predeterminadas. Muy utilizado en GPS y callejeros interactivos.

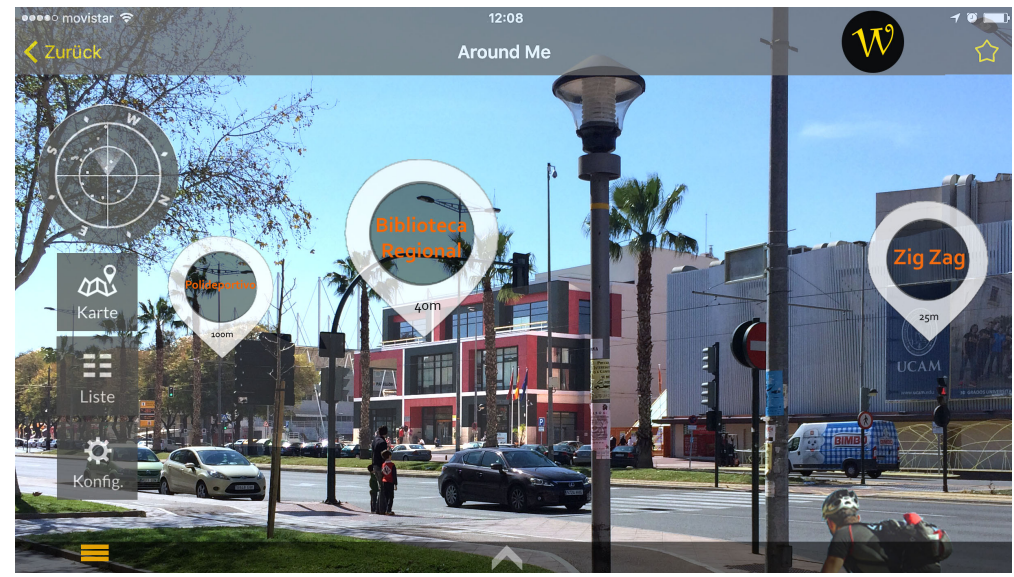

Figura 8. Imagen de un móvil captando mediante una cámara la panorámica de la calle y superponiendo información en tiempo real al "street view" del callejero digital.(Modelo de la aplicación Wikitude).

- Con Software manejado por el usuario (figura 9), requieren mayor atención y creatividad del usuario. Se suelen corresponder con entornos de aplicación académicos o formativos, o de usuarios especializados. 


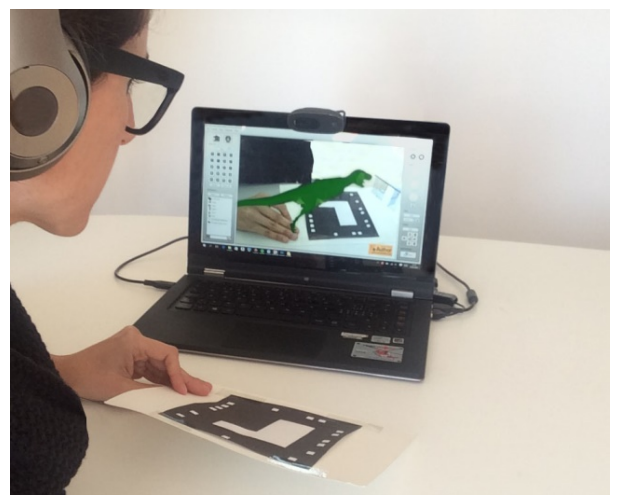

Figura 9. Realidad aumentada generada con el software Aumentaty Author.

- Con interfaces tangibles (figura 10), cuando el objeto está embebido en el entorno real o cuando está dentro de un display responde a señales captadas por sensores. En esta modalidad, un comportamiento intuitivo del usuario genera interacciones con el objeto virtual espontáneas y aparentemente naturales. Requieren un nivel de programación más elevado, pero permite un uso más sencillo e intuitivo. Un campo de aplicación es la RA para niños, para publicidad y marketing, ...
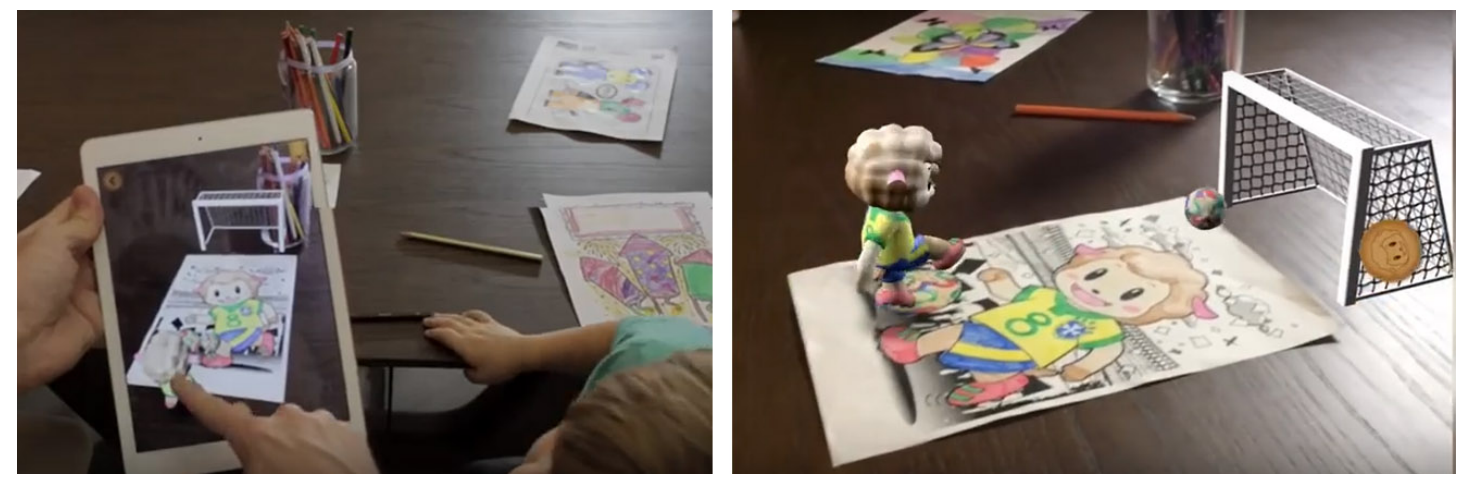

Figura 10. Quiver 3D Coloring (http://quivervision.com/gallery/)

\section{- Los entornos de experiencia RA}

- RA basada en la web (figura 11). Suele ser el modo de usar RA más extendido en educación, marketing, ... Consiste en almacenar unos contenidos previamente configurados y definidos para un propósito que va a tener lugar en espacios concretos y que estarán disponibles en la web para usuarios concretos. 


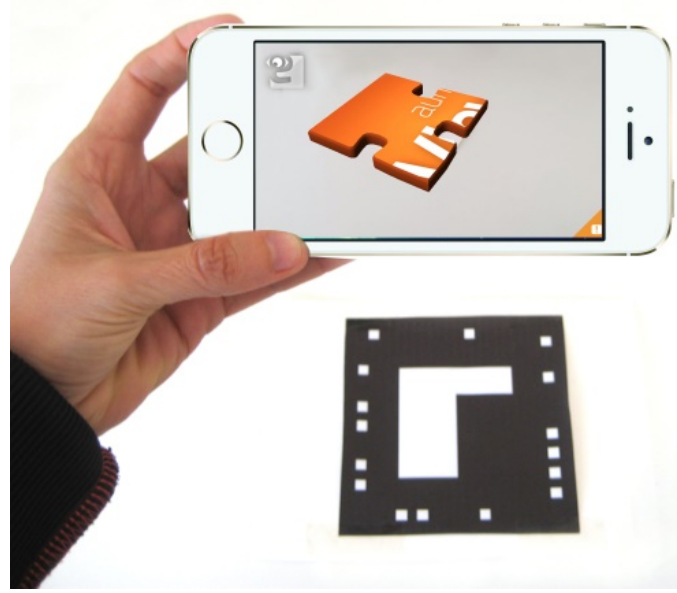

Figura 11. Entorno RA Web-Based. Aumentaty Viewer. (http://author.aumentaty.com/).

- RA móvil en espacios exteriores (figura 12). Es el caso de geolocalizadores acoplados a un „street view“ con información local en 3D en la pantalla del smart phone, gafas AR que reconocen en tiempo real establecimientos, instituciones que estamos viendo en ese momento mientras paseamos por una ciudad,...

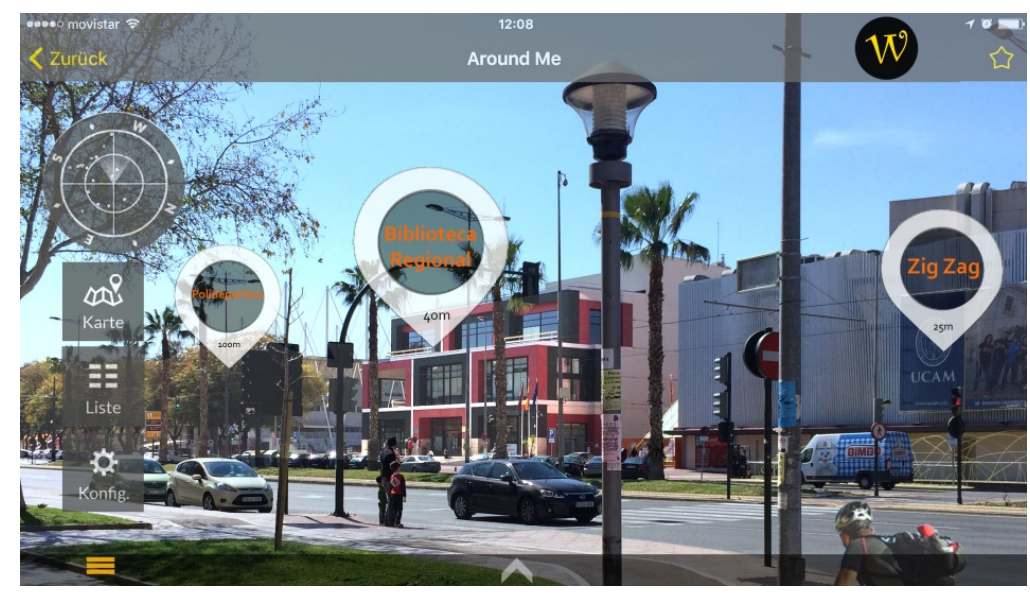

Figura 12. Entorno RA móvil en exteriores. Wikitude.Fotomontaje.

- RA para experiencias localizadas (figura 13). El usuario a menudo no precisa su propio dispositivo, sino que hay una instalación RA preparada en interiores y en exteriores. Es el caso de arte urbano con RA, museos, anuncios publicitarios en la via pública, ... 


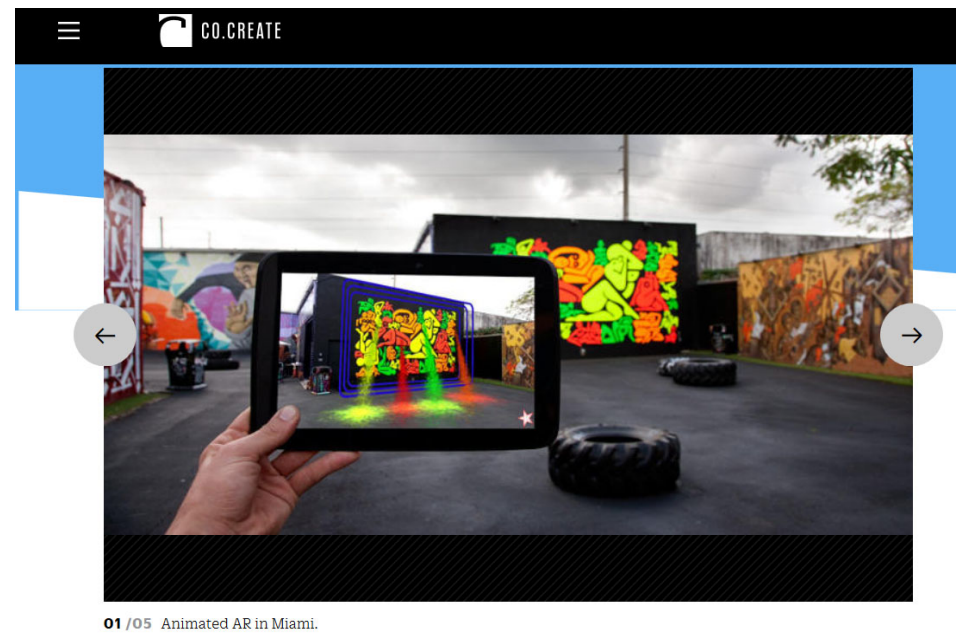

Figura 13. Entorno RA exterior localizado. Proyecto de arte urbano RA,,Rethinking Public Space“, B.C. Biermann. (http://www.fastcocreate.com/1682447/rethinking-public-space-bc-biermann-s-augmentedreality-urban-art).

\section{La tecnología RA como recurso didáctico}

Vamos a suponer que la aplicación de la tecnología RA como recurso didáctico es en principio válida para cualquier asignatura. Nos vamos a situar momentáneamente al margen del proceso de diseño de una actividad didáctica para un área educativa concreta, en la cual el docente haga una selección de un contenido curricular específico y establezca una cohesión interna con un recurso didáctico. Lo que vamos a hacer a continuación es exponer concretamente qué aspectos favorables, basándonos en principios metodológicos, hacen que se proponga la RA, dentro de las tecnologías emergentes, como recurso didáctico aplicable a cualquier asignatura:

\section{- El concepto de "representación del modelo del mundo"}

Cuestiones acerca de cómo preferimos aprender, qué nos gusta más leer, qué asignatura nos motiva más... Investigaciones cualitativas intentan acercarse a una respuesta científica de estas cuestiones de naturaleza subjetiva, que en educación estarían relacionadas con investigar el efecto que la motivación produce en el rendimiento escolar.

Dentro del ámbito de la percepción, estas cuestiones se podrían centrar en el campo de la percepción visual y la representación visual del mundo. Sin embargo "cómo vemos el mundo" es una pregunta de naturaleza subjetiva más compleja que una mera percepción sensorial, una preferencia o un gusto...

Algunas técnicas de comunicación interpersonal intentan acercarse a la complejidad de esta cuestión con el ejemplo del "mundo visual del rinoceronte". Éste siempre ha visto y para él es el mundo "real", un mundo con un cuerno blanco en el centro de su campo visual (figura 14). 


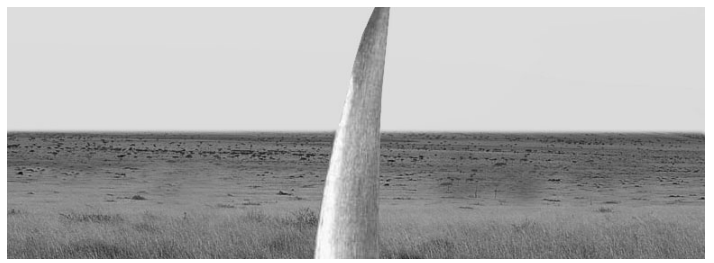

Figura 14. El mundo visto por un rinoceronte.

(http://www.alamany.com/web_kenya/source/oa_ke_00104.htm).

¿Cómo veían el mundo los egipcios? Si tuvieramos que deducirlo de sus representaciones gráficas podríamos afirmar que lo veían en dos dimensiones. La denominada perspectiva egipcia, considera la altura y el ancho según un mismo eje, es decir los egipcios no mostraban en sus representaciones gráficas un indicio estricto de concepto espacial (figura 15). ¿Es así como veían y comprendían el mundo? ¿Es así como intencionalmente pretendían mostrarlo? Las evidencias testimoniales, no responden estas cuestiones, pero si consideramos el dibujo un instrumento de comunicación y representación visual, están comunicando una percepción, comprensión, visión, ... de un mundo en el que una de las superficies del espacio tridimensional es unidimensional.

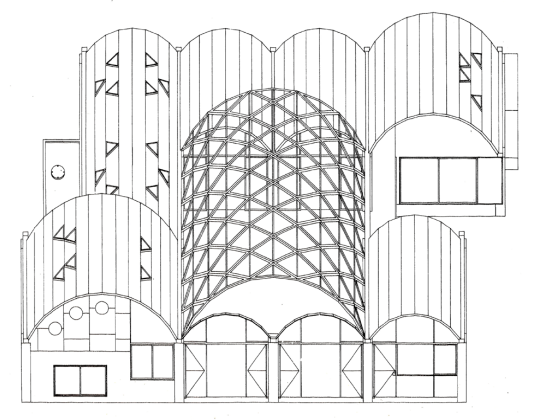

Figura 15. “El lugar reiterado", Metalocus Arquitectura, ITO, TOYO (1988-91). Galería U. Yugawara (Japón). Perspectiva egipcia. @ Toyo Ito \& Associates Architects.

(http://www.metalocus.es/es/noticias/toyo-ito-y-el-lugar-reiterado).

La perspectiva renacentista hace una representación del mundo que enfatiza la profundidad y la posición relativa entre los objetos (figura 16). Se trata por primera vez en la historia de la aceptación generalizada de la representación de una ilusión óptica como nuevo paradigma de la visión del mundo. La perspectiva renacentista es la representación, en una imagen bidimensional, de una percepción ilusoria de la realidad.

La ilusión óptica de Brunelleschi, consistía en fijar la mirada en un único punto de vista, para así observar que líneas paralelas parecían converger hacia un único punto. 


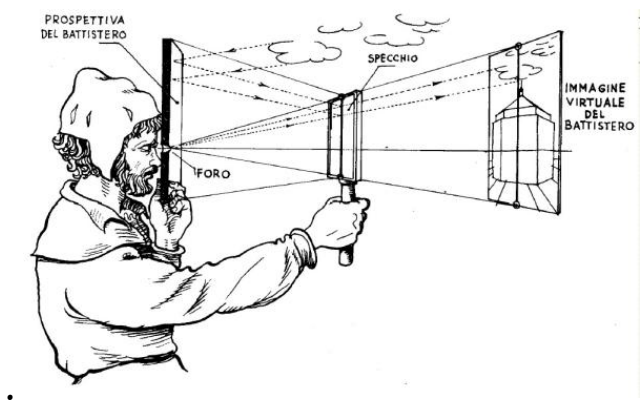

Figura 16. Demostración de la perspectiva lineal con un punto de vista de Brunelleschi. (https://leonardosapprentice.com/tag/augmented-reality/).

En la era digital las técnicas de representación asistidas por ordenador han dado lugar a la representación gráfica, de modo sencillo, de formas muy complejas. Tal es el caso de la representación de superficies curvas NURBS (figuras 17 y 18).

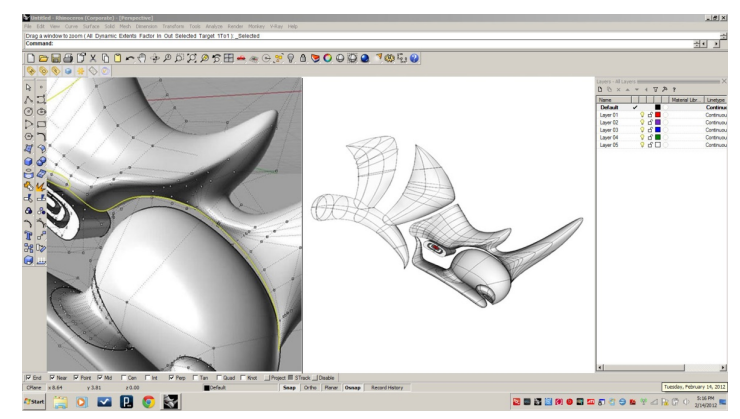

Figura 17. Rhinoceros NURBS design software. (https://www.rhino3d.com/).

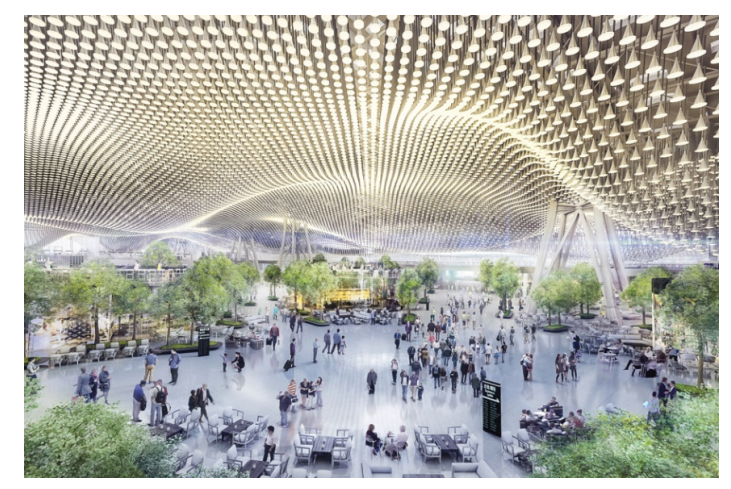

Figura 18. Taoyuan International Airport Terminal 3, in Taiwan, 2016. Proyecto.

ARUP.(http://www.arup.com/news/2016_01_january/22_january_arup_appointed_for_taiwan_taoyuan_ international_airport_terminal_3).

El desarrollo de la representación del mundo en el transcurso histórico ha ido cambiando, de modo coherente con el paradigma de la época. Por otro lado, el paradigma tecnológico y digital actual impregna ya el mundo social, profesional y académico.

La dimensión de lo virtual ha impregnado nuestra cultura actual de la imagen, las técnicas de representación asistidas por ordenador, el cine 3D, los videojuegos, la posiblidad de modelar con ordenador geometrías que antes no eran posibles, ... La RA propone dentro del mundo actual la representación visual de un modelo nuevo de realidad: por medio del 
avance tecnológico se añade la dimensión virtual dentro de un entorno real tridimensional con el cual se interactúa (figura 19).



Figura 19. Modelo de RA colaborativo.

- $\quad$ El valor de la "experiencia vivida" para la mejora del aprendizaje (figura 20)



Figura 20. Pirámide de aprendizaje de Edgar Dale (Flores Cueto, Domínguez Jara, \& Rodríguez Vila, 2010).

En educación las pedagogías constructivistas proponen un aprendizaje basado en la construcción del alumno de su propio aprendizaje y vinculando éste a su experiencia personal, a su vida cotidiana. Las metodologías activas de aprendizaje basadas en la resolución de problemas, en el desarrollo de proyectos personales, parecen responder mejor a este paradigma. Las metodologías de enseñanza para la comprensión, también proponen la experiencia como método para el aprendizaje.

La RA es una tecnología que permite una metodología de aprendizaje-enseñanza basada en estos principios: 
La realidad aumentada introduce de modo visible el conocimiento que el alumno ha de aprender dentro de su entorno real. La abstracción del nuevo conocimiento es visible y está en el entorno físico real del alumno. La experiencia está fundamentando el aprendizaje.

Esta nueva "realidad del proceso de aprendizaje" conlleva una experiencia nueva, de aprendizaje, frente a otros recursos. Además el factor novedad, el factor tecnología emergente y el factor "realidad" pueden suponer un aumento en el nivel de comprensión del alumnado, en la efectividad del proceso de aprendizaje y en la motivación por aprender (Esteban, Restrepo, Trefftz, Jaramillo, \& Álvarez, 2004).

Así como el pedagogo alemán van Hiele (1986) expone su pedagogía para la comprensión en las enseñanzas de matemáticas y geometría, el pedagogo estadounidense Edgar Dale (1934), ya exponía las ventajas de las enseñanzas activas apoyadas en el cine. Edgar Dale se apoyaba en la apreciación que se producía al ver una película de cine y proponía métodos de aprendizaje basados en ver videos, haciendo un uso didáctico de la industria del cine estadounidense por aquellos años. La industria del cine se adecuaba especialmente para la enseñanza debido al desarrollo por aquellos años de las "culturas juveniles". La importancia que para los adolescentes tenía la construcción de su identidad a partir de su imagen, estaba fundamentada en su identificación con la "imagen" proveniente de los "mass media". Este hecho originado por las artes visuales y los canales mediáticos visuales, fue propuesto con fines pedagógicos para la enseñanza secundaria.

La eficiencia del aprendizaje por medio de videos está hoy día asumida. El aprendizaje de determinados contenidos y las plataformas de aprendizaje a distancia y online basadas en videos como material didáctico para el aprendizaje, es una práctica común actualmente en todas las universidades del mundo.

La apropiación por parte del alumno de la producción mediática como experiencia de aprendizaje junto a la construcción de un aprendizaje, pueden integrarse en una experiencia RA.

La interiorización de la experiencia mediática, por medio de la visualización e incluso la producción propia de material mediático, que incluya contenidos curriculares, puede tener potencial suficiente para mejorar el proceso de aprendizaje. Constituye una metodología de aprendizaje activo, para la comprensión, basada en el uso de producciónes mediáticas clave de la era digital.

El uso en secundaria de la tecnología digital y mediática, no intenta fomentar la cultura juvenil de la imagen para fomentar una economía basada en el consumo. Todo lo contrario, proporciona una formación consciente usando estas metodologías y sus recursos, y prepara al alumnado frente a los riesgos que el uso descontrolado de esta tecnología puede suponer para los adolescentes en la creación de las denominadas culturas juveniles de las sociedades de consumo. Siguiendo a las pedagogías que proponen metodologías activas de aprendizaje, la RA es un recurso didáctico que propone una dinámica activa y una actividad pura del alumnado mientras aprende (Flores Cueto, Domínguez Jara, \& Rodríguez Vila, 2010).

La interacción en tiempo real entre el alumno y el contenido curricular en forma de objeto 3D virtual, como aportación metodológica innovadora y singular de la tecnología RA

Acabamos de hablar del valor que añade la experiencia al proceso de aprendizaje, cuando esta forma parte de la metodología didáctica. Se produce una mejora, en términos de 
rendimiento y asimilación, por el mero hecho de que el alumnado aprende por medio de una experiencia personal, que hemos llamado actividad pura.

Hemos presentado esta actividad pura como actividad sensorial: aprender viendo y oyendo el objeto de aprendizaje. Es decir, se trata de desarrollar una metodología basada en el principio de la intuición (utilizando medios audiovisuales principalmente, aprender con experiencias a través de los sentidos). Pero sobre todo la hemos fundamentado en el principio de actividad: aprender haciendo y produciendo resultados visibles, audibles, escribiendo un trabajo, haciendo un proyecto, debatiendo y, en nuestro caso, haciendo una representación escénica con el objeto de aprendizaje.

Pues bien, es en este principio pedagógico de actividad, al que vamos a añadir provisionalmente el calificativo de "actividad escénica" en la que la RA aporta un factor innovador fundamental. Como hemos dicho al principio de este artículo, la tecnología RA está construida sobre tres pilares: intervienen un dispositivo display y un objeto 3D; y se procuce una interacción con el usuario. Vamos a planteamos entonces, de modo desglosado, al margen del alarde tecnológico que la RA es, dónde está su aportación innovadora en el proceso de enseñanza-aprendizaje. Por un lado, la generación de objetos $3 \mathrm{D}$ virtuales no se puede considerar una innovación por sí sola. Multitud de programas y aplicaciones generan objetos 3D y están hechos para un espectro muy amplio de perfiles de usuarios: principiantes, avanzados y expertos, amateurs, académicos y profesionales. En cuando a objetos 3D y el ámbito de la educación, ya hemos comentado también algunas pinceladas históricas de la representaciones 3D. Luego, digitales o análogos, no podemos justificar en el uso de objetos 3D por sí solos, una innovación, ni en general ni en el ámbito de la eduación.

El uso de la tecnología display tampoco se puede calificar de innovadora es sí misma. Esta tecnología sin duda ha experimentado un avance vertiginoso en los últimos años y añade continuamente alguna novedad tecnológica puntual (plana, de plasma, LED, HDR, retina), pero es una pantalla; el invento se hizo a principios-mediados del siglo XX y hoy día hay pantallas en los televisores, los teléfonos, los coches, los electrodomésticos, los ordenadores. Como hemos señalado previamente, el recorrido histórico de las pantallas, el cine, los videos es más reciente. En educación, tiene una cierta componente de novedad la creación de videos, de video tutoriales, video conferencias, video tutorías. Estas están integradas hoy día en cualquier programa de formación online y, aunque tal vez no tanto en las escuelas de formación presencial, todo alumno hoy día recurre a videotutoriales online y a vídeos como recurso didáctivo efectivo. Por lo tanto, tampoco estamos considerando el uso de un display una innovación.

¿Qué hay entonces de la tecnología interactiva? ¿Es una innovación una interacción en tiempo real? Usuarios, docentes, alumnos y profesores-alumnos (en formación continua como profesores), ¿cuándo hemos realizado un aprendizaje con medios interactivos de este perfil? ¿Cuándo hemos usado en nuestra vida cotidiana, académica o profesional un objeto digital 3D interactivo? Al margen de experiencias singulares de carácter individual, esta tecnología tiene un recorrido histórico significativamente más breve. La tecnología multimedia interactiva podríamos aproximar que se inicia hacia 1985. Desde el punto de vista de la comunicación, podríamos decir que se basa en el principio de la comunicación interactiva entre el usuario y el contenido. Es decir, en un proceso interactivo se produce una retroalimentación, una comunicación bidireccional entre el usuario y la máquina, en el cual la máquina "reacciona" en tiempo real a una acción del usuario sobre ella. Nosotros estamos considerando para nuestra propuesta didáctica con RA, un nivel de interacción en el cual se puede transformar el objeto virtual por medio de variables, que el usuario puede modificar. Aquel contenido en el que el alumno tiene mayor dificultad para comprender o 
aquel contenido en el que quiere profundizar, lo puede modificar interactuando con él de acuerdo con sus propias necesidades de aprendizaje. Esto es sin duda un aspecto de gran potencial para las metodologías didácticas: el alumno aprende activamente con un objeto 3D virtual que está dentro de su realidad y de su entorno físico; además lo puede transformar girándolo, modificando su tamaño, seccionándolo según su propio proceso individual de aprendizaje. El alumno no estará viendo un video, estará dentro de una escena que él mismo está transformando: un escenario de aprendizaje interactivo.

Aplicar un recurso innovador suele conllevar un cierto esfuerzo formativo. Si además se trata de una tecnología emergente, que está aún en desarrollo, este factor puede hacer al docente no considerar la RA como un recurso plausible. La idea de que para usar la RA son necesarios conocimientos informáticos avanzados o una formación tediosa en tecnologías aplicadas, puede ser también un motivo de rechazo de la RA, que se prentende modificar con la propuesta didáctica que se presenta a continuación. Así como el uso de una pizarra digital interactiva puede aprenderse por parte del docente en unas pocas horas, el uso de la RA también tiene algunas modalidades de aprendizaje sencillo. Asímismo, con esta propuesta didáctica se intenta evaluar el nivel de competencia digital del alumnado de secundaria y comprobar si el aprendizaje del uso de la RA es un proyecto de aprendizaje realizable en un tiempo razonable.

\section{Experiencia didáctica basada en la aplicación de la RA}

El diseño y elaboración de esta experiencia práctica de enseñanza-aprendizaje se ha realizado siguiendo una metodología activa y de trabajo cooperativo. El alumno ha de crear "un producto RA" en grupos de dos o tres alumnos, reflexionando sobre el concepto RA y utilizando la tecnología RA.

Para la creación del ejercicio didáctico, se ha desarrollado un contexto específico tipo webquest por medio de un blog de aula: una web desde la que se gestiona el ejercicio. La RA se presenta al estudiante dentro de un entorno digital e integrado en un conjunto de recursos TIC: la web en sí, video animaciones, video tutoriales, link externos a otras webs, acceso al blog desde el moodle del centro docente, ... El blog es accesible desde cualquier dispositivo móvil: ordenador, tablet, teléfono móvil. Se ha creado tipo blog para que el alumno escriba comentarios, preguntas, sugerencias,... y que sus compañeros de clase puedan leer también esos "posts"(figuras 21 y 22). De este modo aprenden, moderados por el docente, el uso correcto de las redes sociales.

La creación de la web se ha hecho con la versión gratuita de wordpress. Una de las potencialidades de esta herramienta es su facilidad de uso, facilitando solventar posibles limitaciones de conocimientos del profesorado en el campo de la creación web. Igualmente, wordpress ofrece tutoriales para aprender de modo sencillo a crear webs propias. 


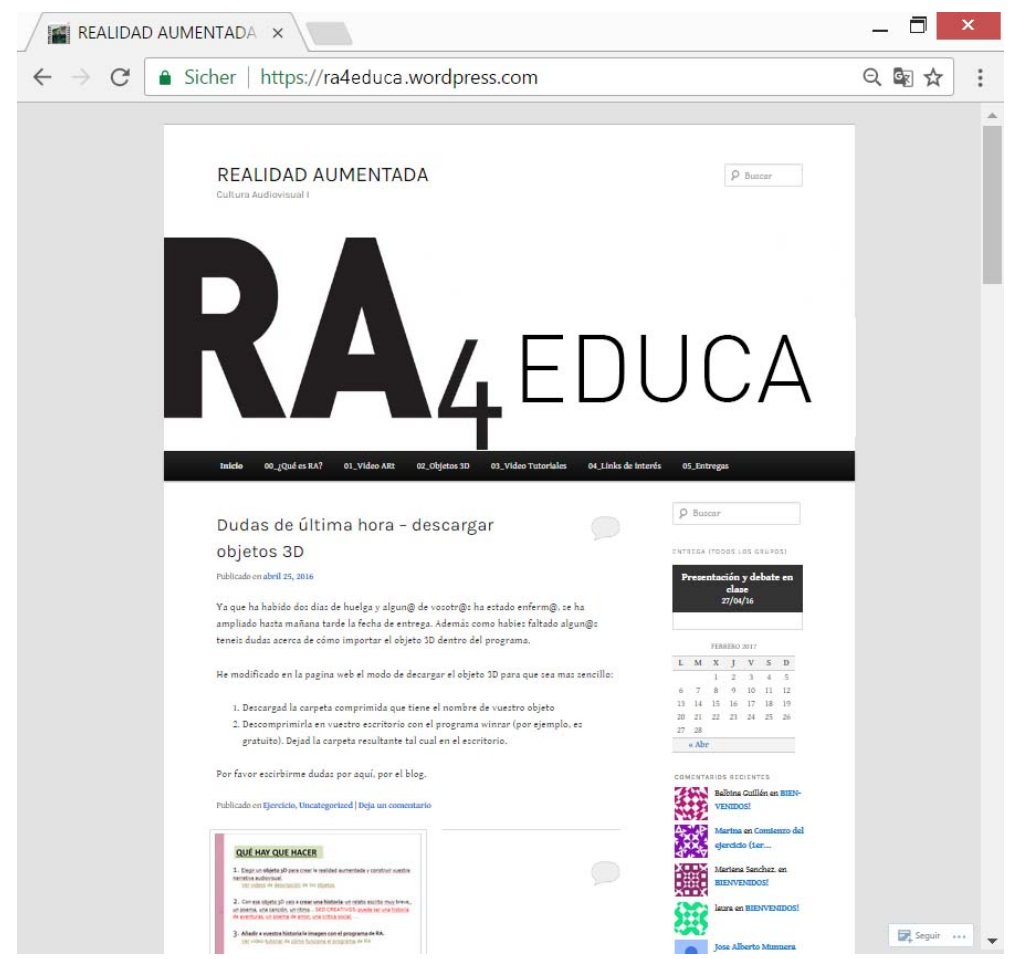

Figura 21. Web tipo blog de aula del ejercicio con RA. Interfaz del alumnado.

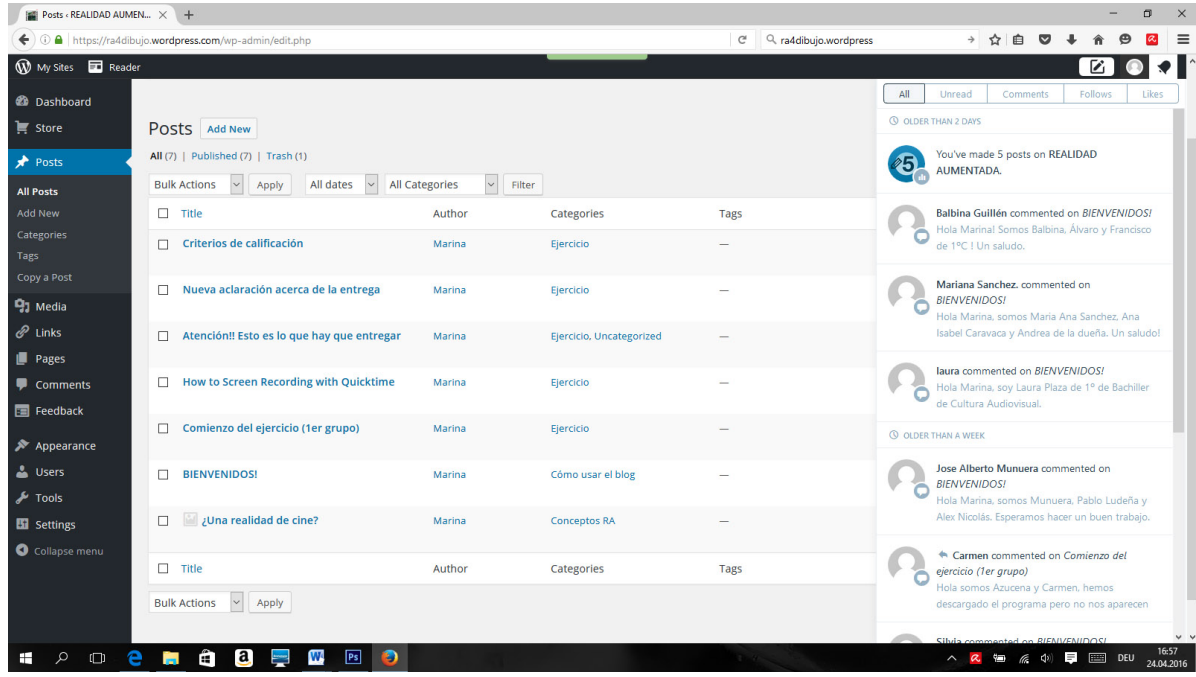

Figura 22. Gestión del foro por el docente desde el blog wordpress del ejercicio.

El diseño de la web está basado en un menú horizontal con página de inicio que contiene el blog de comentarios donde los alumnos pueden escribir sus "posts" y cinco páginas donde se incluye el material didáctico necesario para poder realizar el ejercicio. Tiene por tanto una estructura estándar de fácil acceso y uso. Además se han incluido unas widgets sencillas, tales como una "cuenta atrás" indicando cuanto tiempo queda para que el ejercicio finalice y un calendario. Cada página está encabezada por una imagen o texto tipo eslogan que representa el contenido de la misma. Estos elementos pretenden un entendimiento rápido de cómo deben usar la web. 
La experiencia de aprendizaje consiste en elegir un objeto 3D dentro de un listado de veinte objetos, para crear una escena de RA de temática libre que sea de interés del alumno. EI objetivo de esta actividad es que el alumno se habitúe al uso de tecnología avanzada y al mundo digital de un modo organizado y que entienda cómo está estructurado para un fin concreto. Al tiempo, aprende a usarlos para desarrollar su creatividad y competencia digital. Es una creatividad centrada en la comprensión del entorno físico real en el cual el alumno incluye un objeto 3D de naturaleza virtual para desarrollar igualmente su capacidad de comprensión espacial. Las competencias que se intentan desarrollar en el alumno son la competencia digital (CDIG), la conciencia y la expresión cultural (CEC) y aquella relacionada con el contenido curricular que se esté aprendiendo.

El evaluación del ejercicio será por medio de la escenificación "en directo" de la escena RA o de la grabación de la misma, hecha por el alumno.

Para la realización del ejercicio se han intentado elegir softwares gratuitos y componentes hardware standard:

- $\quad$ Ordenador (portátil o de sobremesa) con cámara integrada o webcam externa

- Programa de realidad aumentada gratuito: aumentaty author (solamente es necesario registrarse online para poder descargar, instalar e utilizar el software). Según el nivel de conocimientos previos y la edad del alumnado, aumentaty ofrece software de diferentes grados de dificultad: author, viewer, ... Además el ejercicio se puede plantear en distintos grados de dificultad.

\section{Materiales didácticos creados para la experiencia de enseñanza-aprendizaje RA, gestionados desde el blog de aula}

Para la implementacion de este ejercicio de aprendizaje se han elaborado materiales didácticos específicos: los recursos didácticos RA (figura 23) y una biblioteca de objetos 3D (figura 24) tomados de repositorios abiertos online.

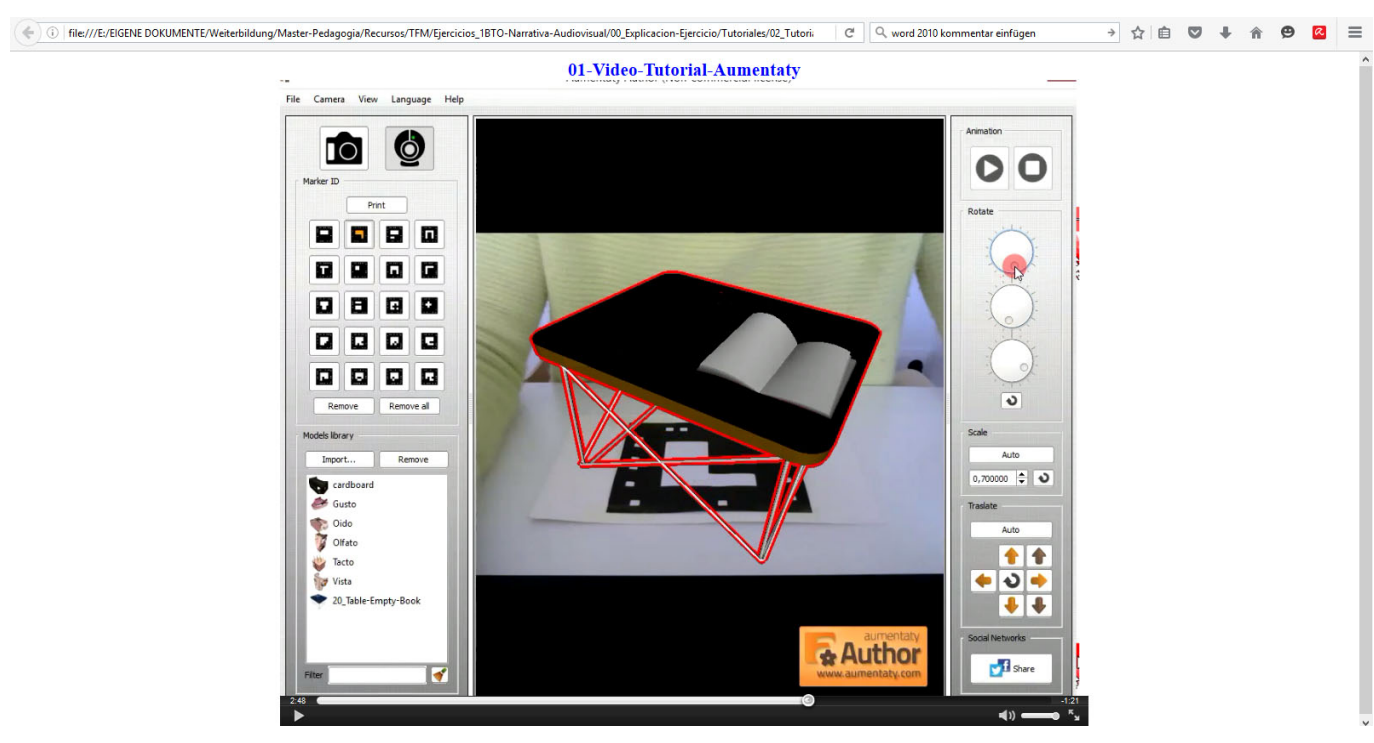

Figura 23. Ejemplificación de un objeto en Realidad Aumentada 

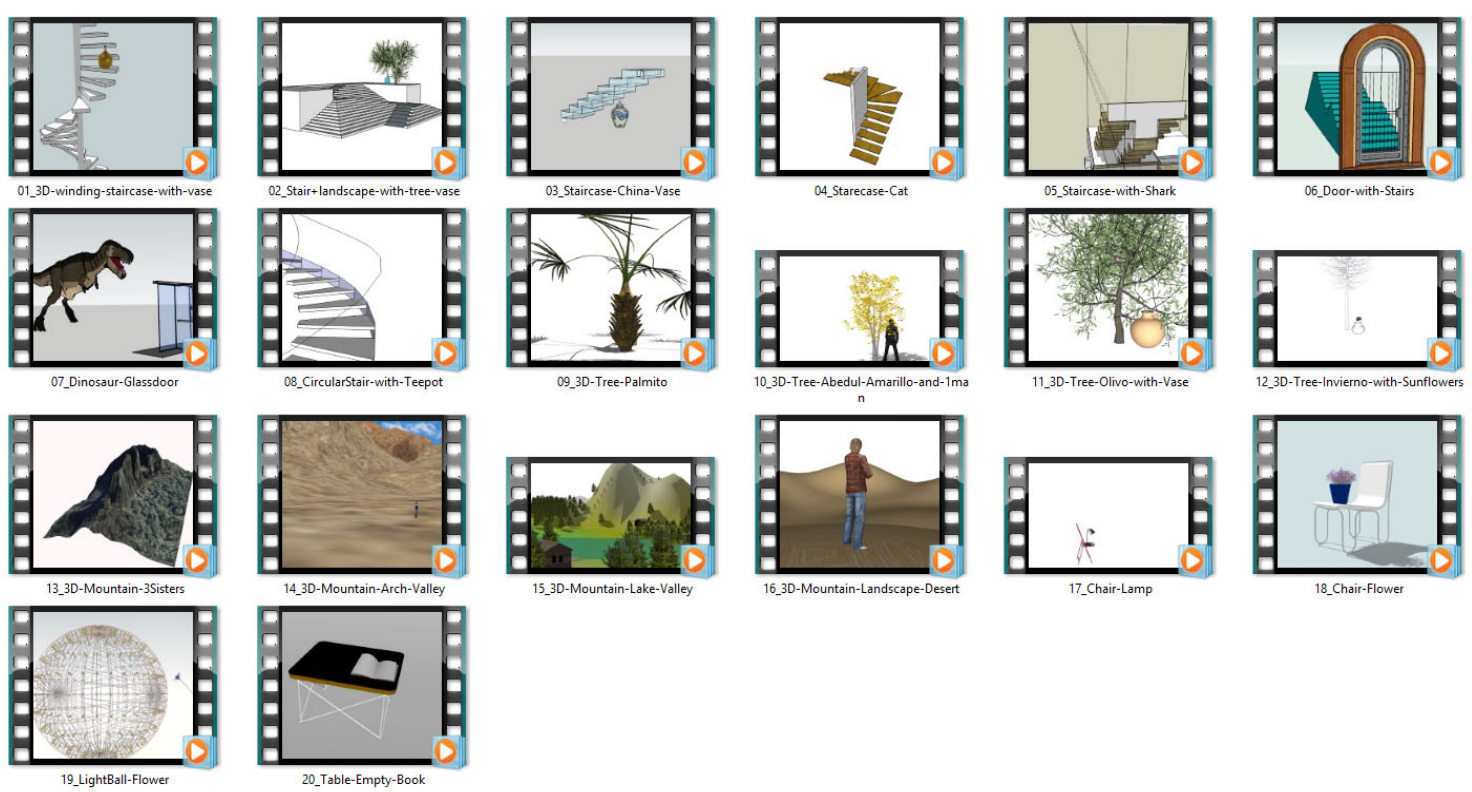

18_Chair-Flower

Figura 24. Material didáctico del ejercicio muestra: biblioteca de objetos 3D para previsualizar como animación y luego visualizar en realidad aumentada.

El repositorio online desde el que se ha creado esta biblioteca específica para este ejercicio es 3D Warehouse de Google SketchUp, donde hay un gran número de objetos 3D, libres y gratuitos, descargables directamente como "Collada File" (figura 25), que es una extensión de archivo que pueden leer los software de RA como Aumentaty Author. Los objetos 3D se podrían haber descargado directamente por el alumno desde 3D Warehouse. No obstante, puesto que es un primer ejercicio con RA, los objetos han sido previamente seleccionados por el docente y se descargan desde la web del ejercicio. Además para facilitar al alumno el inicio del ejercicio, los objetos 3D se pueden previsualizar a modo de animación de unos pocos segundos desde la web. Esta previsualización de los objetos, ha sido igualmente elaborada por el docente para este ejercicio a modo de material didáctico.



Figura 25. Repositorio online de modelos 3D de Google SketchUp (https://3dwarehouse.sketchup.com/?hl=de). 
Según las habilidades del docente, la preparación del material didáctico variará y se podrá ajustar mejor a la diversidad del alumnado y al contenido curricular cuanto más ámplias sean las competencias digitales del docente.

De este modo los diferentes apartados o páginas del webquest del ejercicio, pretenden por un lado describir el ejercicio y por otro lado, adaptarse a diferentes niveles de conocimientos previos del alumnado, faltas de asistencia accidentales y/o posibles errores de comprensión de la actividad en clase. Por estos motivos, consideramos que también pueden ser de utilidad los video tutoriales que explican el concepto RA, la presentación de la tarea, la guía de instalación del software requerido, así como un último apartado de material complementario en el que se ofrecen al alumno contenidos adicionales que enriquezcan y/o amplíen la actividad.

\section{Discusión y conclusiones}

La RA se adecúa a ser aplicada en educación por su potencial para acercar al alumno a una mejor comprensión de los contenios por medio del marco tecnológico e innovador que conlleva y por sus características innatas relativas a la experiencia interactiva y tridimensional del espacio. Tal como hemos desarrollado en este ejemplo de aplicación de realidad aumentada mediante la producción propia o como usuario de esta tecnología, el alumno accede a producciones mediáticas multimedia, al uso de objetos 3D virtuales, a la comprensión espacial, ... La era digital actual ha desarrollado sistemas innovadores de representación de la realidad basados en tecnología software para la creación de modelos $3 \mathrm{D}$ virtuales. Esta realidad está siendo vivida por el alumnado actual fuera de las aulas en videojuegos, películas de cine 3D, programas de ordenador que usarán cuando lleguen a las enseñanzas superiores, al mundo laboral, en su vida,... La realidad aumentada propone acercar este mundo digital a las aulas como recurso didáctico para una educación innovadora.

Las múltiples variantes de esta tecnología permiten adaptar su aplicación en las aulas al nivel de conocimientos previos y competencias adquiridas del alumnado, el profesorado, el equipamiento tecnológico del centro, ... El uso de esta tecnología está además vinculada a medotologías activas de aprendizaje y al paradigma actual de aprendizaje por compentencias. La aplicación de esta tecnología genera una experiencia, en la cual se va a usar un instrumento tecnológico para interactuar en un nuevo concepto de realidad por medio de objetos e informaciones digitales. Por otro lado, la realidad aumentada es idónea para plantear actividades dentro de entornos digitales más ámplios, como webs, redes sociales, foros, ... De este modo, tal como hemos mostrado aquí, toda la gestión del material didáctico se presenta de modo digital para dar a los alumnos acceso a una realidad mixta, la realidad aumentada. En esta nueva realidad, en los objetos digitales 3D añadidos a la realidad física de nuestro entorno, se crea un nuevo espacio donde el alumno accede a la experiencia de aprendizaje, tal como muestra nuestro ejemplo. En ese nuevo espacio mixto, lo virtual puede dar acceso al descubrimiento de un nuevo conocimiento, a crear conocimiento, a una experinecia nueva motivadora, a la visualización de un espacio imaginado y fantástico,... La RA posibilita estas experiencias de aprendizaje de carácter ubicuo, innovador y con tecnologías avanzadas a los alumnos, dentro incluso de las infraestructuras tecnológicas estándar de las escuelas actuales.

El grado de sofisticación e innovación de esta tecnología puede propiciar aumentos en la motivación de los alumnos. Este tema queda abierto para posibles investigaciones posteriores. Ya que la motivación genera aumentos del rendimiento escolar, ¿por qué no ahondar en la cuestión de cómo la innovación educativa por medio de este recurso 
didáctico aplicado, puede mejorar el rendimiento del alumnado? Ya que la realidad aumentada es una tecnología emergente, ¿por qué no acompañar su desarrollo actual con investigaciones acerca de su aplicación efectiva en educación?

Además del potencial que presenta para investigadores en educación, debido a ser un campo abierto de desarrollo e investigación activa actual, como consecuencia de las ramificaciones en el desarrollo de la realidad aumentada, ésta marca una amplia visión de futuro entorno a sus posibles aplicaciones. Arte, cine, arquitectura, ingeniería, matemáticas, psicología, publicidad, marketing, ocio y entretenimiento, educación,... son algunos ejemplos. Debido a su uso en diversas áreas de conocimiento y ámbitos tecnológicos, en diferentes niveles de interacción y de complejidad, etc, las previsiones de uso de esta tecnología pueden extenderse ampliamente en todos los ámbitos de la educación, desde infantil hasta la formación universitaria y de posgrado. Además es un recurso idóneo, igualmente por su multiplicidad de aplicaciones, para el desarrollo de proyectos transversales entre varias asignaturas: tecnología-expresión artística, narrativa-dibujo, inglés, cultura audiovisual, ... El ejercicio presentado en esta propuesta podría diseñarse como actividad de carácter transversal de expresión audiovisual y lenguaje, por ejemplo. Además planificado para una plazo de tiempo mayor, podría ser integrado dentro de una experiencia de aprendizaje por proyectos, integrando planteamientos transversales, que den acceso al alumno a una experiencia de aprendizaje marcadamente innovadora y de carácter integral y ubicuo, propio de los paradigmas actuales en educación.

\section{Bibliografía}

ActivePresenter (Free Edition, Version 5.5.5), Screen Recording \& Interactive e-Learning Authoring Tool [Programa de ordenador]. (2015). Atomy Systems, Inc.

Aumentaty Author, Realidad Aumentada [Programa de ordenador]. (2015). Ayelen Solutions.

3D Warehouse y SketchUp (Trial Version 2016), Biblioteca de objetos 3D y programa de modelado 3D. (2016). Trimble Navigation Limited.

Agudelo Toro, A. (10 de Mayo de 2005). Modelo de contexto para realidad aumentada. Revista Universidad EAFIT, 41 (138), 44-64.

Billinghurst, M.: Mobile AR Lecture 2 - Technology. Recuperado de http://de.slideshare.net/marknboo/mobile-ar-lecture-2-technology

Billinghurst, M., \& Kato, H. Collaborative Augmented Reality. Recuperado de http://www.academia.edu/763015/Collabo rative_Augmented_Reality

Cubillo Arribas, J. (2014). ARLE: Una herramienta de autor para entornos de aprendizaje de realidad aumentada. Tesis Doctoral. Universidad Nacional de Educación a Distancia.

Cubillo Arribas, J., Martín Gutiérrez, S., Castro Gil, M., \& Colmenar Santos, A. (2014). Recursos Digitales Autónomos Mediante Realidad Aumentada. RIED, Revista Iberoamericana de Educación a Distancia, 17 (2), 241-274.

Díaz Barriga, Á. (2013). TIC en el Trabajo de Aula. Impacto en la Planeación Didáctica. Revista Iberoamericana de Educación Superior, IV(10), 3-21.

Esteban, P., Restrepo, J., Trefftz, H., Jaramillo, J. E., \& Álvarez, N. (2004). XVI Simposio Iberoamericano de enseñanza Matemática. La realidad aumentada: un espacio para la comprensión de conceptos del cálculo en varias variables. Castellón, España: Universidad Jaime $\mathrm{I}$. 
Flores Cueto, J. J., Domínguez Jara, C. M., \& Rodríguez Vila, J. J. (Edición Nº 10. Julio 2010). La Realidad Aumentada como Herramienta para Mejorar los Procesos Educativos en la USMP. Boletín electrónico de la Unidad de Virtualización Académica (UVA).

Gartner, I., Gartner Research. Recuperado de http://www.gartner.com/technology/research.jsp

Johnson, L., Smith, R., Willis, H., Levine, A., \& Haywood, K., (2011). The 2011 Horizon Report. Austin, Texas: The New Media Consortium. 